\title{
Sharpening of natural guide stars for low-order wavefront sensing using patrolling laser guide stars
}

R. Dekany, C. Neyman, R. Flicker

R. Dekany, C. Neyman, R. Flicker, "Sharpening of natural guide stars for low-order wavefront sensing using patrolling laser guide stars," Proc. SPIE 7015, Adaptive Optics Systems, 701525 (10 July 2008); doi:

10.1117/12.790171

Event: SPIE Astronomical Telescopes + Instrumentation, 2008, Marseille, France 


\title{
Sharpening of Natural Guide Stars for low-order wavefront sensing using patrolling Laser Guide Stars
}

\author{
R. Dekany ${ }^{\mathrm{a}}$, C. Neyman ${ }^{\mathrm{b}}$, and R. Flicker ${ }^{\mathrm{b}}$ \\ ${ }^{a}$ California Institute of Technology, Pasadena, CA 91125 \\ ${ }^{\mathrm{b}}$ W. M. Keck Observatory, 65-1120 Mamalahoa Hwy., Kamuela, HI 96743
}

\begin{abstract}
A laser guide star (LGS) adaptive optics (AO) system generally requires additional tip/tilt information derived using a natural guide star (NGS), while multi-LGS systems will benefit from measurement of additional low-order wavefront modes using one or more NGS's. If we use AO sharpened NGS's, we can improve both the measurement accuracy and accessible sky fraction while also minimizing the observational overhead of faint NGS acquisition. Multi-object adaptive optics (MOAO) sharpening of NGS is possible where a good estimate of the NGS wavefront can be made, for example where tomographic wavefront information is available. We describe a new approach for high Strehl ratio sharpening, based on additional patrolling laser beacons, to eliminate generalized anisoplanatism and minimize tomography error in the NGS direction.
\end{abstract}

Keywords: Adaptive Optics, Laser Guide Star, wavefront sensing

\section{INTRODUCTION}

Current laser guide star (LGS) adaptive optics (AO) systems ${ }^{[1],[2],[3]}$ and those envisioned for the near future ${ }^{[4],[5]}$ require measurement of low-order wavefront modes using available natural guide stars (NGS) selected from the available patrol range or field of regard (FoR). For photon-noise-limited low-order wavefront sensing, the measurement accuracy is optimized for adaptively sharpened $\mathrm{NGS}^{[6]}$. In this case, sensing can be performed using the diffractionlimited core of the AO point spread function (PSF) to significant benefit, assuming sufficient sharpening Strehl ratio.

Multiconjugate AO (MCAO) has been proposed as a method of sharpening field NGS to improve the quality of low-order measurements $s^{[7],[8],[9]}$. Unfortunately, to obtain the highest sky coverage fraction on current and extremely large telescopes (ELT's), we must select field NGS from a field diameter extending over two arcminutes or more. Generalized anisoplanatism ${ }^{[10]}$ limits the ability of simple dual-conjugate MCAO systems to provide good sharpening over such large FoR. As an example, for the median Mauna Kea Ridge atmospheric turbulence profile model ${ }^{[11]}$, having $\mathrm{r}_{0}=16 \mathrm{~cm}$ and $\theta_{0}=2.7 \operatorname{arcsec}$ at $\lambda=0.5 \mu \mathrm{m}$, dual-conjugate correction at optimized deformable mirror (DM) conjugate altitudes of $0.6 \mathrm{~km}$ and $12.2 \mathrm{~km}$ results in wavefront error due to generalized anisoplanatism at a location one arcminute off-axis of $\sim 301 \mathrm{~nm}$ RMS. This single term error alone limits the near-infrared $\mathrm{H}[\mathrm{J}]$-band Strehl ratio for this NGS to $27 \%[10 \%]$. While an off-axis distance of one arcminute would be unusually large for a classical AO system utilizing a single tip/tilt reference star, future systems utilizing multiple low-order NGS will mitigate the otherwise objectionable tilt anisoplanatism error, making wide NGS asterisms interesting when seeking the very highest sky coverage fraction.

Extension of MCAO to three-conjugate correction or more could theoretically improve field NGS sharpening, but this increases the complexity of MCAO system optical design. Maintaining small pupil aberrations while relaying a wide field of view is difficult, particularly for large aperture telescopes ${ }^{[12]}$. The inclusion of additional DM's in series also introduces additional transmission losses and complications in the MCAO control law.

\subsection{MOAO sharpening of field NGS}

Multiobject $\mathrm{AO}^{[13]}$ (MOAO) provides an attractive alternative to MCAO for the purpose of field NGS sharpening. In the MOAO paradigm, a separate deformable mirror is dedicated to each object of interest, allowing wavefront correction calculated specifically for that direction in the sky. Because each target can be corrected individually, MOAO eliminates generalized anisoplanatism error. In exchange, MOAO increases computational hardware requirements, as the tomographic wavefront estimate must be calculated in each target direction. Furthermore, because each DM correction is applied without optical feedback to the high-order wavefront sensor paths, MOAO correction must be

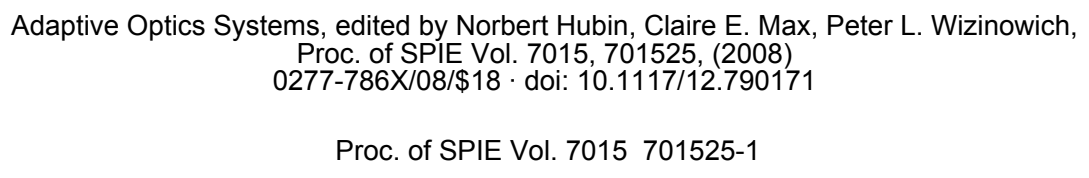


applied using a 'go-to' control law, requiring excellent calibration and stability of all DM's and high-order WFS. The maturity of both DM and WFS calibration has been experimentally verified in the laboratory and on-sky in full MOAO system demonstrations $^{[14]}$. These demonstrations have shown the contribution from new MOAO-specific wavefront error sources, offset by improvements in correction temporal bandwidth, to be approximately $30 \mathrm{~nm}$ RMS due to DM calibration and $50 \mathrm{~nm}$ RMS due to WFS calibration issues ${ }^{[14]}$.

Table 1 summarizes the MOAO benefit for the Mauna Kea Ridge model and assumed 10-meter diameter telescope.

\begin{tabular}{|l|c|c|c|c|}
\hline $\begin{array}{c}\text { AO Mode for } \\
\text { NGS sharpening }\end{array}$ & Error term & $\begin{array}{c}\text { RMS wavefront } \\
\text { error at 60” off- } \\
\text { axis distance }\end{array}$ & $\begin{array}{c}\text { J-Strehl } \\
\text { upper } \\
\text { limit }\end{array}$ & $\begin{array}{c}\text { H-Strehl } \\
\text { upper } \\
\text { limit }\end{array}$ \\
\hline MCAO alone & Generalized anisoplanatism & $301 \mathrm{~nm}$ & $10 \%$ & $27 \%$ \\
\hline MOAO & Go-to control errors (incl. calibration) & $<100 \mathrm{~nm}$ & $>78 \%$ & $>87 \%$ \\
\hline
\end{tabular}

Table 1. Benefit of MOAO sharpening compared to MCAO sharpening alone for a 60 " off-axis field NGS, assuming the Mauna Kea Ridge atmospheric model and a 10-meter diameter telescope. These results pertain only to the wavefront error arising from the difference in how wavefront corrections are applied in the two paradigms. Both MCAO and MOAO approaches would additionally suffer tomography error from imperfect atmospheric sampling (§2).

Of course, MOAO sharpening and MCAO are not mutually exclusive. In fact, the TMT NFIRAOS MCAO system has proposed MOAO sharpening of NGS at infrared wavelengths for low-order wavefront sensing as an upgrade path for the first light facility ${ }^{[15]}$. The relative merit of adding MOAO sharpening to any AO system will depend on the specific science benefit to further improving low-order wavefront sensing sky coverage and the future cost trends for commercial, high actuator count, deformable mirrors and large-format, low-noise wavefront sensors.

\subsection{MOAO sharpening with LTAO}

An alternative approach, being pursued by the W. M. Keck Observatory Next-Generation Adaptive Optics (NGAO) team $^{[16],[17],[18],[19]}$ is to combine MOAO sharpening with pupil-conjugated deformable mirror correction based on laser tomography AO (LTAO). In this hybrid approach, the first LTAO stage applies a relatively low-order correction to the entire field of view, using a moderate format DM in a common pupil plan. Subsequent to this, MOAO correction is applied in the direction of each field NGS, using a dedicated, small physical diameter, large format DM.

The hybrid LTAO/MOAO approach to sharpening field NGS has several advantages. Whereas a classical MOAO system would need to correct both static telescope and atmospheric wavefront errors, the hybrid approach requires MOAO correction of only the anisoplanatic component of the wavefront error. In many cases, this reduces the dynamic range and linearity requirements on the MOAO DM's. Because the high-order WFS light can be made to enjoy this same first stage LTAO correction, feedback on the shared DM shape is provided, forming a quasi-closed-loop system. This additionally eases the requirements on high-order LGS WFS dynamic range and linearity. The hybrid approach does, however, require successful MOAO to enjoy these benefits. The failure of MOAO in this hybrid approach would very significantly compromise available sky coverage.

\section{TOMOGRAPHY ERROR FROM FIXED VS. PATROLLING LGS}

When MOAO sharpening field NGS, the fundamental limiting term in the error budget for NGS sharpening, assuming sufficient signal-to-noise ratio, is tomography error, the ability of the system to accurately estimate the direction-specific correction given uncertainty in the turbulence height, missing wavefront information, and errors arising from blind mode combinations of wavefront errors.

In the NGAO hybrid LTAO/MOAO approach, we seek to optimize the total science target wavefront correction, as a combination of the tomography error on-axis and the residual low-order wavefront sensing error using sharpened field NGS. The on-axis tomography error directly impacts the target sensitivity, while the off-axis field NGS tomography error reduces sharpening and increases low-order mode measurement error, indirectly impacting science target sensitivity. In general, diffraction-limited AO systems are designed to balance the Strehl loss arising from high-order 
and low-order errors. As we look forward to truly ubiquitous AO system usage, we wish to consider the case of highest sky coverage fraction, where overall performance is typically dominated by residual tip/tilt errors.

Fixed LGS asterisms have been proposed for large telescope LTAO and MCAO systems ${ }^{[20],[21],[22]}$. Because good wavefront correction is needed in both the science target direction and in the directions of each of the field NGS used for low-order wavefront sensing, the radii of these LGS asterisms have typically been selected to provide a reasonable compromise between the tomography error on-axis and that across the NGS field of regard.

We propose the use of a reconfigurable LGS asterism to simultaneously provide near-optimal tomographic wavefront sensing for the on-axis science target direction and for each field NGS direction. Intuitively, we expect improved tomographic correction in the vicinity of each LGS, where the tomographic interpolation/extrapolation from the instantaneously measured wavefront directions is minimized. In this approach, LGS light can be portioned into a smaller fixed LGS asterism, optimized for tomography in the on-axis science direction, and a patrolling LGS asterism configured to provide the best field NGS sharpening on a target-by-target basis.

Figure 1 illustrates one possible comparison between the classical fixed LGS asterism, which we refer to as the pentagonal packing asterism, and the proposed hybrid fixed/patrolling LGS asterism for the case of six LGS. In this hybrid case, we have elected to move three LGS inward to provide better on-axis tomography, while reconfiguring three LGS to point in the hypothetical direction of three NGS. Based on previous studies ${ }^{[23],[24]}$ investigating tomography error vs. asterism radius for a fixed geometry, we select from the class of all possible pentagonal packing asterisms one having a radius of 50". Generally, this radius is near optimal for six LGS, as any larger radius pentagonal packed asterism begins to suffer increasing on-axis tomography error, and increasing azimuthal variations in tomography error for large off-axis evaluation directions.

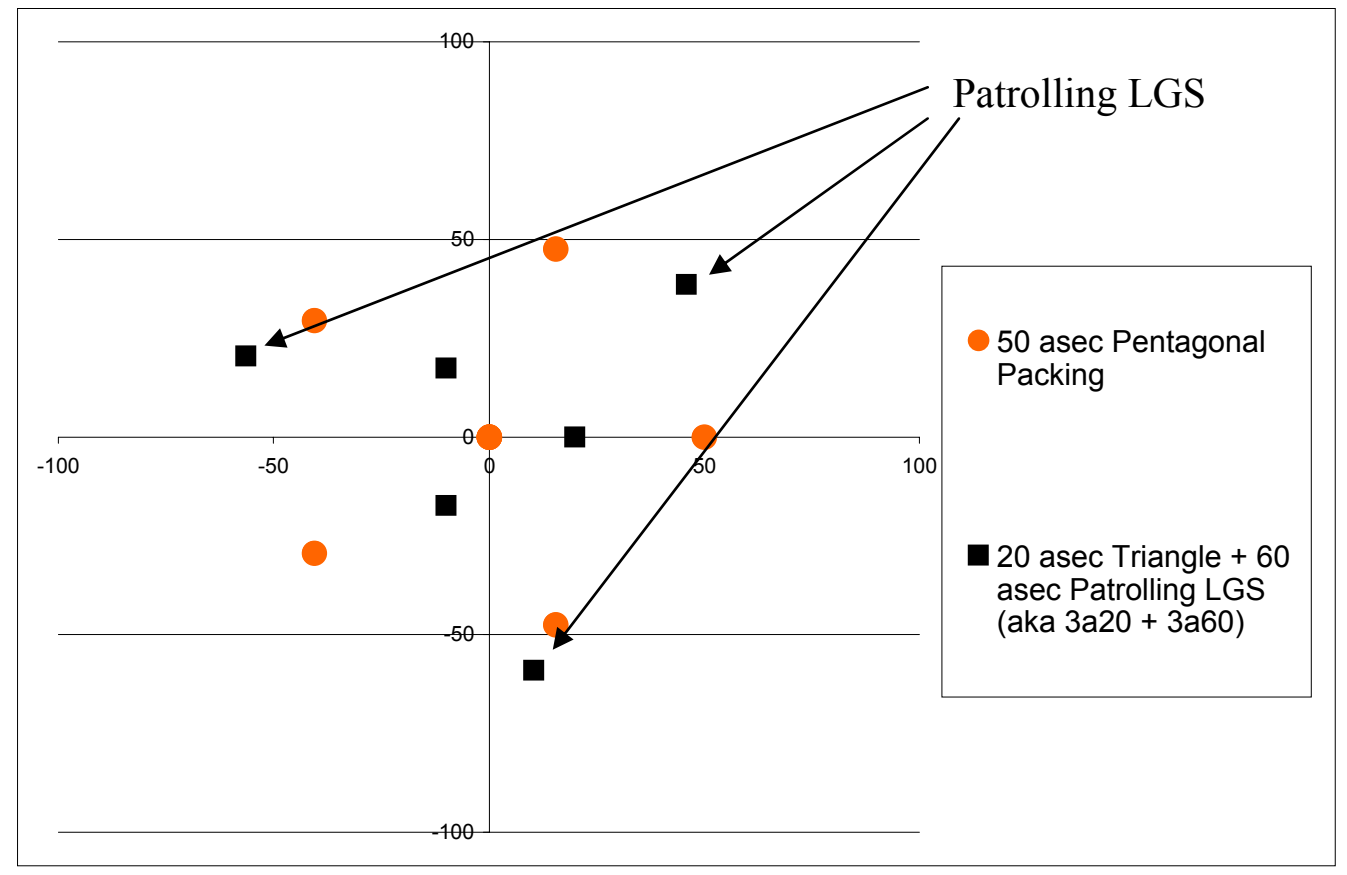

Figure 1. Example asterisms for this study. The 50" radius Pentagonal Packing asterism has a central LGS and a regular pentagon of LGS having 50" radius. The hybrid fixed/patrolling asterism has an unoptimized, fixed 20" radius triangular inner asterism and a reconfigurable outer asterism, here chosen to be a 60 " radius triangle rotated arbitrarily with respect to the inner triangle. The outer asterism is intended to correspond to a typical high-sky-fraction NGS asterism, with a patrolling laser targeting each NGS. 
To explore the relative merit of fixed vs. patrolling LGS asterism, we evaluated the tomography error for the two asterisms shown in Figure 1. Simulations in this paper were performed with the LAOS simulation ${ }^{[25],[26],[27],[28]}$ developed by Thirty Meter Telescope project. LAOS is a complete Monte Carlo AO simulation that performs both an explicit tomography step to estimate the volume of turbulence above the telescope and a fitting step that determines the correction to apply based on a user selectable set of evaluation points. In the case of MOAO, i.e. several LGS's and one DM per target, the fitting step is optimized for each target point (science or NGS) independently. In the case of MCAO, i.e. several LGS and a few DM's correcting all field points simultaneously, the correction is based on user selectable weighting to optimize the correction over the field of view using a finite number of deformable mirrors. LAOS simulations were performed that isolate the effect of tomography from other AO system errors. In order to isolate the tomography error, the simulations were noise free and the system was modeled to have an infinite correction bandwidth, that is a zero time delay between measurement and correction of the wavefront.

The spacing of the actuator grid and the size of the subapertures were set to $0.3516 \mathrm{~m}$. This spacing results in approximately 32 subapertures and 33 actuators across the 10-meter diameter Keck Telescope pupil. The atmospheric $\mathrm{C}_{\mathrm{n}}^{2}$ profile and wind speed were the Mauna Kea Ridge model with $\mathrm{r}_{0}=16 \mathrm{~cm}$ and $\theta_{0}=2.70$ arcseconds. Single star NGS simulations with the same phase screen seeds were run to estimate the effect of the fitting error with 32 actuators across the pupil. The tomography error was estimated by taking each LGS run and subtracting the NGS results in quadrature. The results from the five random seeds were averaged to get the final results; each seed was run until the AO loop achieved stable performance. Tomography performance was evaluated at 49 points on a rectangular grid with 20 arc second spacing between points; the boundary of the sample points was a square 120 arc seconds on a side. Other sample points were selected at the location of the laser guide stars and at other key locations located in the outskirts of LGS constellation.

The results of our simulation comparison are shown in Figure 2, which show contour plots representing the tomography error evaluated in different directions, using $10 \mathrm{~nm}$ RMS contours beginning at $60 \mathrm{~nm}$ RMS. The tomography error as a function of off-axis radius is shown in Figure 3.
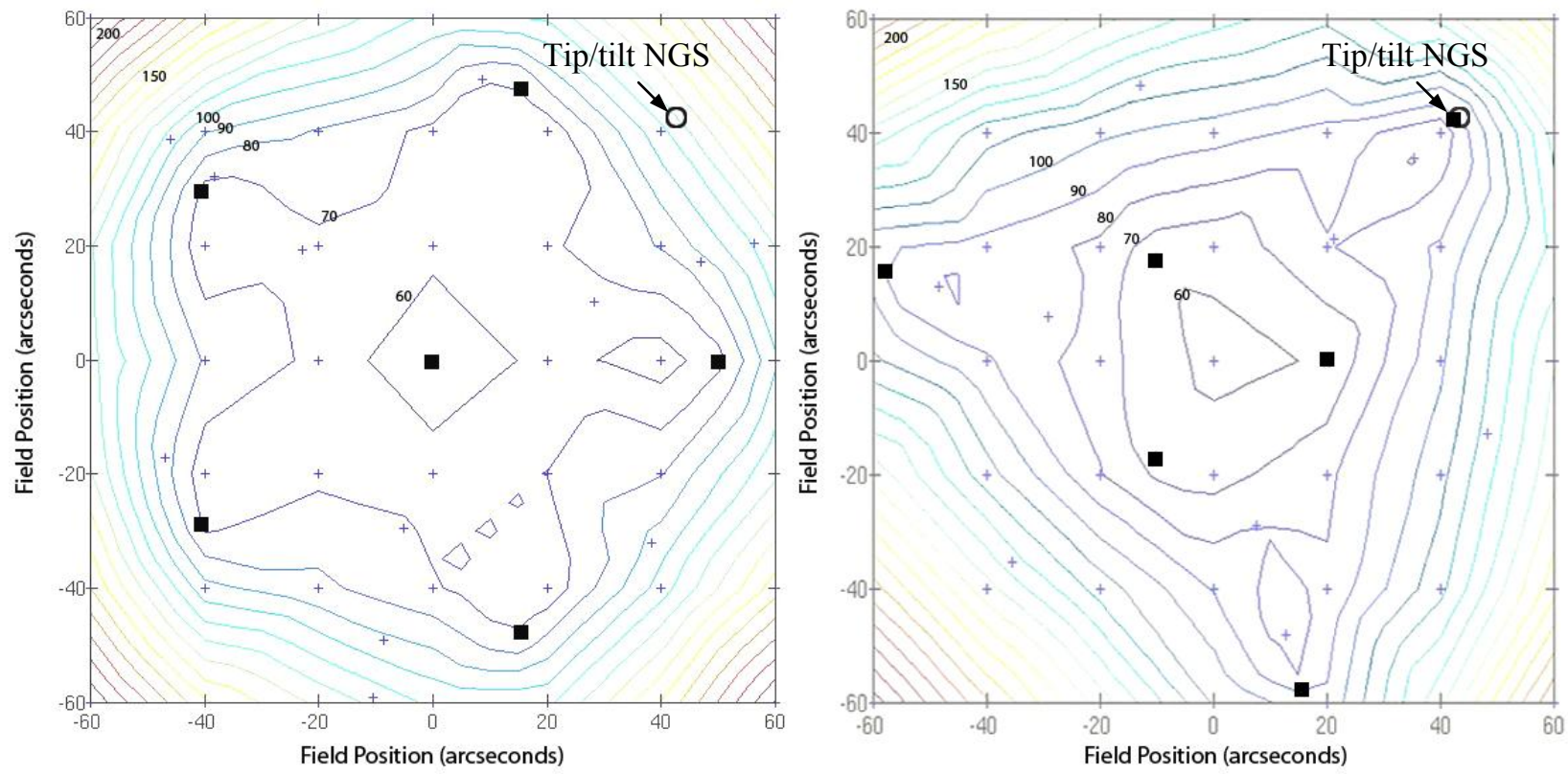

Figure 2. Comparison of the tomography error for the 50" Pentagonal Packing asterism (left) and the 20" Triangle +60 " Patrolling LGS asterism (right) shown in Figure 1. In the direction of a hypothesized 60" off-axis NGS (indicated by the open circle) the patrolling asterism provides lower tomography error. Pentagonal packing asterisms of larger radii exhibit increasingly objectionable azimuthal variations for NGS at large off-axis distance. Evaluation points are indicated by the small plus symbols. 


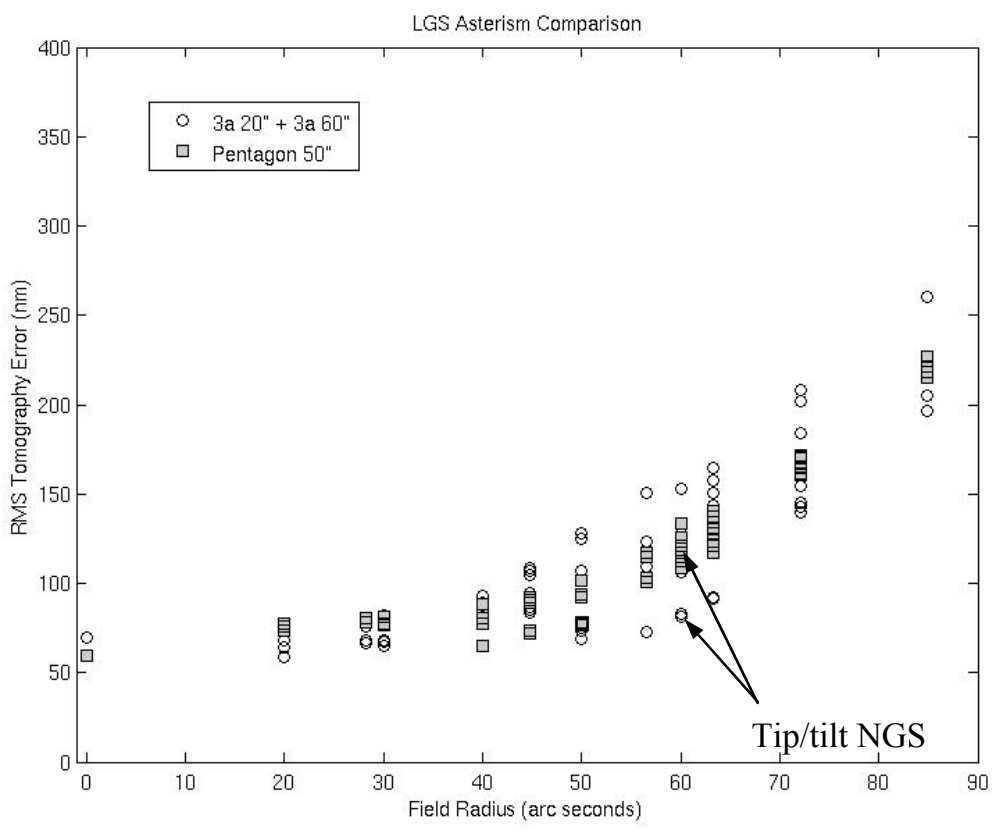

Figure 3. Tomography error at evaluation points indicated in Figure 2. The benefit of patrolling LGS is seen at the 60" field radius, where the lowermost open circles represent the tomography error in the field NGS direction.

We find that the hybrid patrolling LGS asterism does indeed enjoy lower tomography error at the field NGS positions that matter for an MOAO sharpening strategy, as summarized in Table 2. We see the benefit of patrolling LGS is a reduction in tomography error from approximately $125 \mathrm{~nm}$ RMS to $95 \mathrm{~nm}$ RMS. When considered in the context of a full NGS sharpening error budget, containing for example $150 \mathrm{~nm}$ RMS wavefront error from all other sources, the relative benefit of patrolling LGS is somewhat reduced, providing J [H]-band Strehl of 45\% [63\%] vs. 38\% [58\%] for the fixed asterism. Although these results only apply directly to the fixed 50" radius pentagonal packaging asterism, we have found similar results at other radii, with larger radii pentagonal packing asterisms beginning to unduly compromise the on-axis tomography error in the science target direction (see for example reference [24]).

\begin{tabular}{|l|c|c|c|}
\hline \multicolumn{1}{|c|}{ LGS Asterism } & $\begin{array}{c}\text { RMS tomography } \\
\text { wavefront error in the } \\
\text { three NGS directions }\end{array}$ & $\begin{array}{c}\text { J-Strehl } \\
\text { upper } \\
\text { limit }\end{array}$ & $\begin{array}{c}\text { H-Strehl } \\
\text { upper } \\
\text { limit }\end{array}$ \\
\hline Pentagonal packing with 50" radius & $\begin{array}{c}110-140 \mathrm{~nm} \\
(125 \mathrm{~nm} \text { mean })\end{array}$ & $67 \%$ & $80 \%$ \\
\hline $\begin{array}{l}\text { Inner 20" radius triangle and three } \\
\text { patrolling LGS pointed directly at } \\
\text { field NGS }\end{array}$ & $95 \mathrm{~nm}$ & $80 \%$ & $88 \%$ \\
\hline $\begin{array}{l}\text { Inner 20" radius triangle and three } \\
\text { patrolling LGS at optimum location } \\
\text { (see §3.) }\end{array}$ & $80 \mathrm{~nm}$ & $85 \%$ & $91 \%$ \\
\hline
\end{tabular}

Table 2. Summary of tomography error in the NGS direction of fixed and hybrid patrolling LGS asterisms. The indicated Strehl ratios correspond to that from tomography error alone.

We should note that the fixed 50" pentagonal packing asterism provides slightly lower tomography error at around 40 " radius, particularly near the LGS themselves, but there is no benefit to this since there are, by assumption, no field NGS there to sharpen. In a practical observation, if one or more NGS were available at smaller off-axis radii, the patrolling LGS asterism would be similarly adjusted inward to once again provide the best tomography in the field NGS 
directions. For systems requiring low sky coverage fraction, where the rare availability of multiple appropriate NGS within 30 " radius suffices, patrolling LGS are found to lose their advantage, as the 50" pentagonal packing asterism provides excellent tomography throughout it's deep interior.

Of course, a more typical distribution of NGS in any given science field will consist of NGS of widely varying offaxis distance. The selection of any given fixed asterism will result in poor MOAO sharpening of the outermost NGS, or alternatively somewhat degraded sharpening of all NGS.

\section{OPTIMIZED PATROLLING LGS PLACEMENT}

We are further interested in knowing, for any given field NGS off-axis distance, what is the optimum direction to which to point a patrolling LGS to affect the greatest MOAO sharpening, through reduction of tomography error. Intuitively, we expect greater fractional sensing of the atmospheric cylinder encountered by the field NGS light if we were to point a patrolling LGS in a direction slightly radially outward from the LGS position (so that the optical marginal ray from the LGS object moves to lie atop the marginal ray from the science target.) For a 10-meter telescope, this extra radial angle is 11.5 arcseconds outboard of the field NGS, for a $90 \mathrm{~km}$ distant beacon.

Working against this potential benefit, the absolute value of tomography error interior to the patrolling asterism could well grow with increasing asterism. If the absolute value of tomography error in the field NGS direction were to grow when pointing outside the field NGS, it would defeat the potential benefit of this strategy.

To investigate the situation, we conducted detailed LAOS simulations comparing two noise-free LGS asterisms that differ only in the off-axis radius of their patrolling LGS component. Contour plots of the corresponding tomography errors are shown in Figure 4, where the gray squares indicate the alternative LGS asterism geometries. The contours shown are based upon the approximately rectilinear evaluation grid indicated by the shown plus symbols.
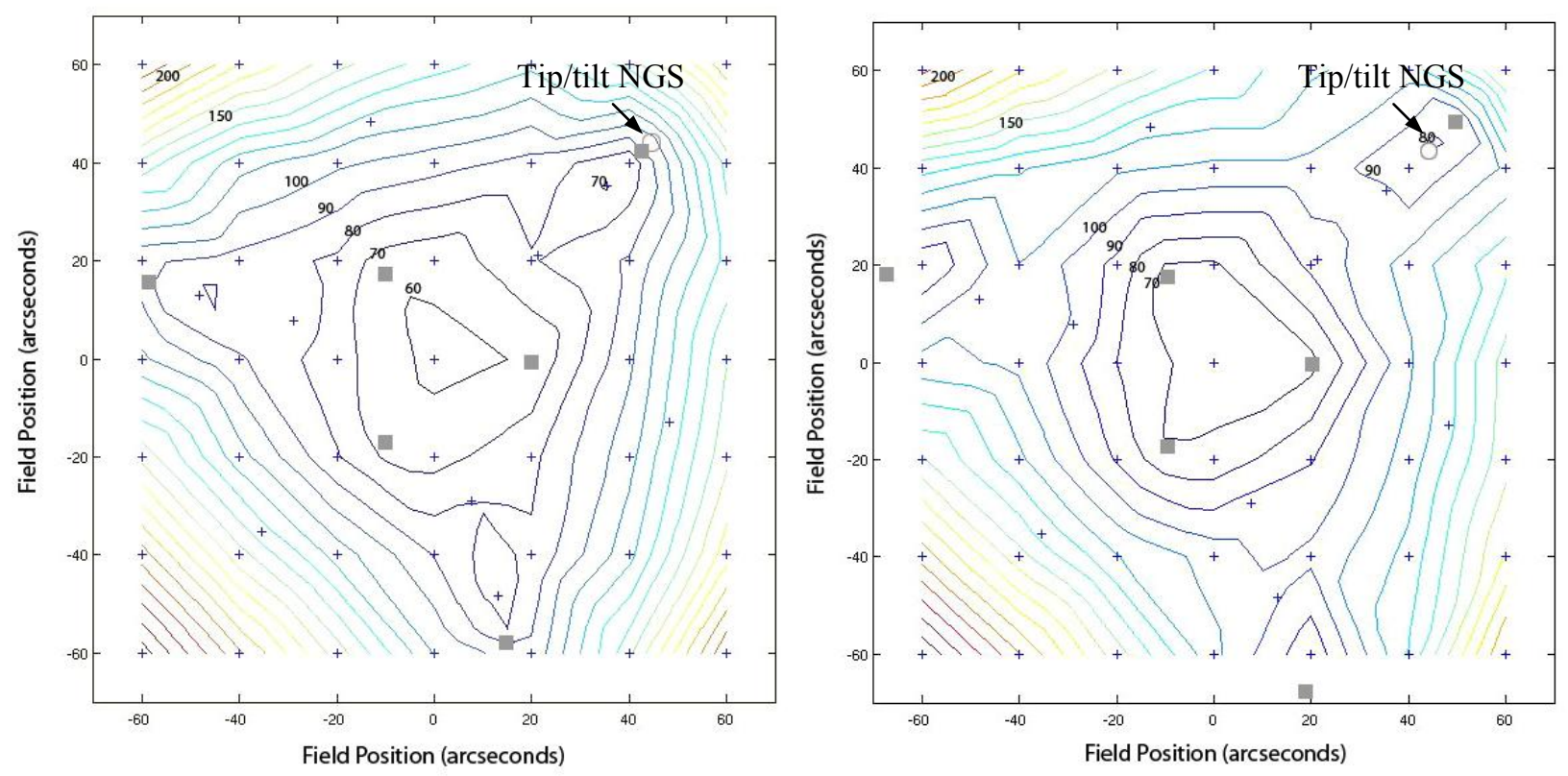

Figure 4. Noise-free RMS wavefront tomography error for asterisms of 3 x 20 " fixed LGS in addition to 3 x 60 " patrolling (left) and $3 \times 70$ " patrolling (right) LGS. LGS locations are indicated by gray squares. Contour interval is $10 \mathrm{~nm}$ RMS. An example field NGS location is indicated by the open circle in upper right of each plot. At this location, the 70" LGS asterism results in $80 \mathrm{~nm}$ RMS wavefront tomography error, whereas the 60" LGS asterism results in $100 \mathrm{~nm}$ RMS. Better NGS sharpening, therefore, is obtained by pointing the patrolling LGS about 7" outside the field NGS location.

As seen in Figure 4, the tomography error for a typical field NGS (indicated by the open circle) is indeed minimized (both locally and absolutely) by pointing the patrolling LGS several arcseconds outside the nominal field NGS location. 
For the example here, the tomography error contribution at the open circle location is decreased from $95 \mathrm{~nm}$ RMS to $80 \mathrm{~nm}$ RMS, even though the patrolling asterism is increased from 60 " to 70 " radius. The improvement in field NGS sharpening gained through this technique corresponds (c.f. Table 2) to about $5 \%[3 \%]$ increased $\mathrm{J}[\mathrm{H}]$-Strehl from this term alone, or 3\% [3\%] increased $\mathrm{J}[\mathrm{H}]$-Strehl when this term is root-sum-squared with an additional $150 \mathrm{~nm}$ RMS wavefront error from all other sources. While the specific benefit of pointing outside the field NGS is rather small in absolute terms, radial off-pointing is likely to also provide a more stable correction compared to pointing the LGS directly at the NGS.

It should be noted, that the 70" patrol asterism does exhibit a slight (about $6 \mathrm{~nm}$ RMS) degradation in on-axis science target tomography error relative to the 60 " patrol asterism, as the inner fixed 20 " asterism is not optimized to maintain the on-axis performance. Strictly speaking, the strategy of pointing patrolling LGS slightly outside the field NGS should only be considered when the benefit of better field NGS sharpening outweighs the science target tomography degradation. In the case of performance limited by tip/tilt errors due to measurement noise of the field NGS positions, the $18 \%$ improvement in field NGS J-Strehl arising from optimum patrol placement more than compensates the small increase in on-axis tomography error.

In our simulations we found that the optimum radial LGS offset beyond a 50" off-axis field NGS is about 11 arcseconds, while the corresponding optimum distance beyond a 60 " off-axis NGS is approximately 7 arcsec. As the field NGS off-axis distance is increased further, we would expect the optimum offset distance would continue to decrease until the off-axis distance becomes so large that the tomographic benefit of all other LGS in the asterism is lost. In that limit, we expect that the patrolling laser should once again be pointed directly at the field NGS. In other words the minimum wavefront sensing error should revert to the case of a single LGS suffering classical focal anisoplanatism (FA) error at large off-axis NGS distance. This feature of patrolling LGS is not present with classical fixed asterisms, where wavefront errors will continue to grow with increasing off-axis distance. Importantly, far off-axis NGS suffering even full focal anisoplanatism error will still typically enjoy stronger sharpening with MOAO than would be obtained with MCAO correction which suffers ever-increasing generalized anisoplanatism error.

For decreasing off-axis field NGS radial distance, on the other hand, we would expect the optimum LGS pointing offset distance to continue grow until a field NGS radius is reached beyond which there is no advantage of LGS patrolling at all. At this point, all field NGS within some limiting radius would enjoy nearly the same excellent tomographic wavefront sensing from a fixed LGS asterism. Although we did not venture to find this 'inner edge', our results in $\$ 2$ indicate that for a total of six LGS, there is little advantage of patrolling LGS at off-axis distances less than about 40 arcseconds.

As an important caveat, we point out that our simulations have so far only considered patrolling LGS all having equal off-axis distance. In reality, any viable patrolling LGS strategy would need to be verified considering actual asymmetric patrolling asterisms. We expect our basic conclusions will continue to hold for asymmetric patrols, under the conditions simulated (6 LGS with a field of regard of 150 arcseconds on a 10-meter diameter telescope.)

\section{GENERAL CONSIDERATIONS FOR METAPUPIL SHEAR}

In order to satisfactorily sample the entire atmospheric cylinder in the science target direction, and thus overcome classical focal anisoplanatism error, an LGS asterism outer radius must scale approximately linearly with telescope diameter. As this asterism radius is increased to overcome focal anisoplanatism (FA) for extremely large telescopes (ELT's), good tomographic information will be naturally available over large fields of regard. Thus, the relative advantage of patrolling LGS over fixed LGS asterism will be diminished. This can be interpreted in terms of the corresponding metapupil shear ${ }^{1}$.

Excellent tomography solutions can generally be found for metapupil shears large enough to provide effective resolution of the three-dimensional turbulence profile, but not so large that the corresponding on- and off-axis pupil projections separate, resulting in an objectionable component of error from missing wavefront information. Where offaxis NGS would suffer large metapupil shear and thus missing information, the use of patrolling LGS can restore the tomographic solution in the NGS direction, without compromising the on-axis solution. ELT's used with modest LGS asterism radius can enjoy good vertical resolution for sensing with relatively small metapupil shear.

\footnotetext{
${ }^{1}$ We define the metapupil shear as the fractional relative offset of the entrance pupil, as projected onto a given atmospheric turbulence layer for an off-axis object field location, relative to the entrance pupil projection onto the same layer for an on-axis field point.
} 
In the converse case, smaller aperture telescopes have a more difficult time properly sampling a large pseudofrustum of atmosphere. Because of this, the component of tomography error due to incomplete sampling of the atmospheric volume is relatively large. Patrolling LGS can be positioned optimally in order to minimize the unsampled volume of atmosphere in the NGS direction.

Extending this rationale, the merit of patrolling LGS would also be enhanced for atmospheric $\mathrm{C}_{\mathrm{n}}^{2}(\mathrm{~h})$ profiles having smaller isoplanatic angles, because the relevant metapupil shear in a given off-axis direction grows proportionately with effective turbulence height. Although all our simulations to date have assumed multiple sodium LGS systems, based upon metapupil shear considerations, we would similarly expect the relative advantage of patrolling LGS over fixed asterism systems to be enhanced in Rayleigh LGS systems utilizing relatively few beacons.

When the density of LGS on the sky is large, there is only a small metapupil shear between the MOAO direction and multiple nearby LGS. Thus, although we have only simulated here asterisms containing 6 sodium LGS for 10-meter telescopes, we expect that the merit of patrolling LGS is rapidly diminished with increasing number of LGS beacons. In fact, earlier results ${ }^{[23]}$ indicate that 9 optimally-placed fixed sodium LGS can provide uniformly excellent wavefront estimation over a FoR of 60 " radius, eliminating the benefit of patrolling LGS at the cost of increased LGS projection, wavefront sensing, and laser cost and complexity.

Thus, the regime of greatest applicability for the patrolling LGS strategy will be for mid-sized telescopes ${ }^{2}$ needing large sky coverage fraction in conditions of small isoplanatic angle. In this demanding case, excessive metapupil shear thwarts the accuracy of fixed-asterism tomography over fields large enough for sufficient natural star counts.

\section{PRACTICAL CONCERNS}

The proposed performance advantages of patrolling LGS, however, also come at a price. Several practical drawbacks exist and must be considered in weighing the decision to implement patrolling LGS in any real system.

\subsection{Optomechanical complexity}

The patrolling LGS strategy requires increased optomechanical complexity in both the projection system to generate a reconfigurable laser asterism and in the acquisition for the similarly patrolling high-order wavefront sensors. It should be noted, however, that patrolling LGS are similar in complexity to strategies utilizing fixed asterism geometry but variable asterism radius. Such a capability may be desired within precision AO systems to allow the LGS asterism to be continuality 'trimmed' to optimize tomography error as the atmospheric vertical turbulence profile changes.

\subsection{Reconstructor generation}

Because each science target field will use a different field NGS asterism, the tomographic wavefront reconstruction algorithm for both science target and field NGS correction would need to be pre-generated based upon good knowledge of the location and suitability of each candidate field NGS. If candidate field NGS are discovered to actually be extended galaxies, a complete new set of reconstructors would need to be generated in quasi-real-time, potentially requiring costly computing resources not needed for fixed LGS asterism strategies.

\subsection{Observational efficiency}

Multiple field NGS systems will inevitably require greater pre-observatory preparation time than current single field NGS systems. The inclusion of patrolling LGS, however, does not require greater observer preparation time. Aside from possible compute overheads, the operational time overhead for patrolling LGS acquisition is not likely to outlast the time required for field NGS acquisition ${ }^{[30]}$ (due to the vastly greater brightness of the LGS). In fact, because the patrolling NGS strategy should optimize the Strehl ratio of the field NGS, overall acquisition times would actually be reduced for a photon-noise-limited acquisition process.

\subsection{Sequencer and system complexity}

The addition of patrolling LGS will inevitably add complexity to an AO system's sequencing tasks. With more degrees of freedom under computerized motion control, more hardware and control routines requiring initialization, a

\footnotetext{
${ }^{2}$ For sufficiently small telescope apertures, FA error is sufficiently diminished to obviate the need for multiple LGS and thus multiple field NGS. For systems requiring only a single tip/tilt or tip/tilt/focus NGS, the principle of patrolling LGS could still be employed using a second LGS in the NGS direction along with MOAO compensation.
} 
greater universe of system states including fault states, and more documentation required, the overall effort required to implement patrolling LGS might very well be more effectively deployed for any given project addressing other, performance limiting factors such as maximizing return LGS photosignal or minimizing WFS noise equivalent centroiding error.

\section{CONCLUSIONS}

We have proposed a new paradigm for measurement of low-order atmospheric wavefront aberrations, the use of patrolling laser guide stars, to provide improved tomographic information in order to optimize MOAO sharpening of NGS. We find that when considering a goal of sharpening 60" off-axis NGS, utilizing LGS asterisms of 6 sodium beacons on a 10-meter diameter telescope, assuming a typical Mauna Kea $\mathrm{C}_{\mathrm{n}}{ }^{2}(\mathrm{~h})$ profile, patrolling LGS's can provide up to $18 \%$ absolute J-band Strehl ratio advantage considering tomography error alone, and typically $10 \%$ absolute Strehl advantage when considered as part of a realistic field NGS sharpening error budget.

Patrolling LGS's appear to be of particular advantage for current medium and large aperture telescopes demanding the highest sky coverage fraction in conditions of small isoplanatic angle, where the corresponding tomography error from fixed LGS asterism is objectionable at large off-axis distance. Even so, the practical considerations of increased system complexity indicate that patrolling LGS are suitable for only the most demanding high-sky-coverage AO applications.

\section{ACKNOWLEDGEMENTS}

This research was carried out in support of the W. M. Keck Observatory Next Generation Adaptive Optics System preliminary design phase. We wish to acknowledge support for this effort from the National Science Foundation through the Telescope System Instrumentation Program (TSIP) and thank the technical staff at TMT for their support of the LAOS simulation code.

\section{REFERENCES}

[1] Wizinowich, P., et al., "Adaptive Optics Developments at Keck Observatory," SPIE Proc. 6272, 627209, (2006).

[2] Roberts, J., et al., "Facilitizing the Palomar AO Laser Guide Star System," SPIE Proc. 7015, 7015-99, (2008).

[3] Fesquest, V., et al., "Gemini North laser guide star system: operations, maintenance, and. science observations review," SPIE Proc. 7015, 7015-226, (2008).

[4] d'Orgeville, C., et al., "The Gemini South MCAO Laser Guide Star Facility: getting ready for first light," SPIE Proc. 7015, 7015-96, (2008).

[5] Hackenberg, W., et al., "Four-laser guide star facility for the VLT adaptive optics facility," SPIE Proc. 7015, 7015102, (2008).

[6] Rigaut, F. and Gendron, E., "Laser guide star in adaptive optics. The tilt determination problem," Astron. Astrophys. 261, 677-684 (1992).

[7] Beckers, J. M., "Increasing the size of the isoplanatic patch size with multiconjugate adaptive optics", in ESO conference on Very Large Telescopes and their instrumentation, M.H. Hulrich, ed., pp. 693, (1988).

[8] Ellerbroek, B., "First order performance evaluation of adaptive optics system for atmospheric turbulence compensation in extended field-of-view astronomical telescope", J. Opt. Soc. Am A 11, pp. 783-805, (1994).

[9] Arcidiacono, C., et al., "Sky coverage for Layer Oriented MCAO: a detailed analytical and numerical study", Proc. SPIE 5490, pp. 563-573.

[10] Tokovinin, A., and Viard, E., "Limiting precision of tomographic phase estimation." Journal of the Optical Society of America A 18, 873-881, (2001).

[11] Dekany, R., "Mauna Kea Ridge Turbulence Models", Keck Adaptive Optics Note \#503, W. M. Keck Observatory, Kamuela, HI (2007).

[12] Bauman, B., "Anisoplanatism in adaptive optics systems due to pupil aberrations," Proc. SPIE 5903, pp. 236-247 (2005).

[13] Gendron, E., et al., C. R. Physique, 6 (2005). 
[14] Gavel, D., et al., "Visible light laser guidestar experimental system (Villages): on-sky tests of new technologies for visible wavelength all-sky coverage adaptive optics systems," SPIE Proc. 7015, 7015-15, (2008).

[15] Herriot, G., et al., "NFIRAOS: TMT narrow field near-IR facility adaptive optics," Proc. SPIE 6272, pp. 62720Q (2006).

[16] Wizinowich, P., et al., "W. M. Keck Observatory's next-generation adaptive optics facility," Proc. SPIE 7015, 7015$36,(2008)$.

[17] Max, C., et al., "The Science Case for the Next Generation AO System at W.M. Keck Observatory," SPIE Proc. 7015, 7015-225, (2008).

[18] Le Mignant, D., et al., "Science Operations for NGAO at the System Design Phase," SPIE Proc. 7015, 7015-010, (2008).

[19] Gavel, D., et al., "Concept for the Keck next-generation adaptive optics system," SPIE Proc. 7015, 7015-226, (2008).

[20] Angel, J. R. P. and Lloyd-Hart, M., "Atmospheric tomography with Rayleigh laser beacons for correction of wide fields and $30 \mathrm{~m}$ class telescopes," Proc. SPIE Conference on Adaptive Optical Systems Technology, ed. P. L. Wizinowich, 4007, 270-276, (2000).

[21] Ellerbroek, B. L., et al., "MCAO for Gemini-South," in Adaptive Optical System Technologies II, (Proc. SPIE), eds. P. L. Wizinowich \& D. Bonaccini, 4839, 55 (2003).

[22] Lloyd-Hart, M., et al., "Multi-laser-guided adaptive optics for the Large Binocular Telescope," SPIE Proc. 6691, (2007).

[23] Flicker, R., "LGS asterism geometry and size," Keck Adaptive Optics Note \#429, W. M. Keck Observatory, Kamuela, HI, (2006).

[24] Gavel, D. and Neyman, C., "Tomography Codes Comparison and Validation," Keck Adaptive Optics Note \#475, W. M. Keck Observatory, Kamuela, HI, (2007).

[25] Gilles, A. L. and Ellerbroek, B., "LAOS: Linear Adaptive Optics Simulator", Thirty Meter Telescope Project Office, TMT.AOS.TEC.05.084.DRF01, (2005).

[26] Ellerbroek, B., "Efficient computation of minimum-variance wave-front reconstructors with sparse matrix techniques", JOSA A, 19, 1803-1815, (2002).

[27] Ellerbroek, C. B., Gilles, L., and Vogel, C., "Numerical simulations of multiconjugate adaptive optics wave-front reconstruction on giant telescopes", Applied Optics, 42, 4811-4818, (2003).

[28] Gilles, D. L., "Closed-loop stability and performance analysis of least-squares and minimum-variance control algorithms for multiconjugate adaptive optics", Applied Optics, 44, 993-1002 (2005).

[29] Wizinowich, P., et al., “The W.M. Keck Laser Guide Star Adaptive Optics System: Overview,” PASP 118, 297-309, (2006).

[30] Campbell, R., et al., "AO Operations at the W.M. Keck Observatory,” SPIE Proc. 7016, 7016-002, (2008). 